\title{
Identifying hub genes and potential mechanisms associated with senescence in human annulus cells by gene expression profiling and bioinformatics analysis
}

\author{
CHAO LIU $^{1 *}$, NONG CHEN ${ }^{2 *}$, KAI HUANG ${ }^{3 *}$, MINBO JIANG $^{4}$, \\ HE LIANG $^{1}$, ZHONGYI SUN ${ }^{1}$, JIWEI TIAN ${ }^{5}$ and DEGUO WANG ${ }^{1}$
}

${ }^{1}$ Department of Orthopaedics, The Central Hospital of Songjiang, Shanghai 201600; ${ }^{2}$ Department of Orthopaedics, Qingpu Branch of Zhongshan Hospital Affiliated to Fudan University, Shanghai 201700; ${ }^{3}$ Department of Orthopaedics, Shanghai General Hospital of Nanjing Medical University, Shanghai 201620;

${ }^{4}$ Department of Orthopaedics, Shanghai Fengxian Hospital, Shanghai 201400; ${ }^{5}$ Department of Orthopaedics, Shanghai Jiao Tong University Affiliated First People's Hospital, Shanghai 201620, P.R. China

Received April 20,2016; Accepted March 20, 2017

DOI: $10.3892 / \mathrm{mmr} .2017 .8322$

\begin{abstract}
The aim of the present study was to reveal the potential hub genes and regulatory mechanisms associated with senescence in human annulus cells by analyzing microarray data using bioinformatics. The gene expression dataset GSE17077, of senescent and non-senescent annulus cells obtained from patients with disc degenerative diseases (DDD), was downloaded from the Gene Expression Omnibus database. Differentially expressed genes (DEGs) were identified. Functional and pathway annotations were performed using Gene Ontology and Kyoto Encyclopedia of Genes and Genomes terms, respectively. Web-based Gene Set Analysis Toolkit and Chip Enrichment Analysis were used to identify key transcription factors (TFs). A protein-protein interaction (PPI) network was constructed to analyze the hub genes associated with senescence in DDD. A total of 667 DEGs were screened, including 368 up- and 299 down-regulated genes. These DEGs were enriched in phosphorylation, regulation of apoptosis and regulation of programmed cell death. In addition, DEGs were involved in axon guidance, natural killer cell-mediated cytotoxicity, purine metabolism
\end{abstract}

Correspondence to: Dr Deguo Wang, Department of Orthopaedics, The Central Hospital of Songjiang, 746 Middle Zhongshan Road, Shanghai 201600, P.R. China

E-mail: wangdeguo@live.cn

Dr Jiwei Tian, Department of Orthopaedics, Shanghai Jiao Tong University Affiliated First People's Hospital, 650 New Songjiang Road, Shanghai 201620, P.R. China

E-mail: doctjw@163.com

*Contributed equally

Key words: disc degeneration, senescence, differentially expressed gene, gene expression profiling, bioinformatics and the mitogen-activated protein kinase (MAPK) signaling pathway. The TFs activator protein 1 (AP1), specificity protein 1 and aryl hydrocarbon receptor may serve regulatory roles in gene expression in senescent cells. Certain key target genes of TFs, including heat shock protein 90 (HSP90) and $\mathrm{C}-\mathrm{X}-\mathrm{C}$ motif chemokine 5 (CXCL5), within the DEGs were revealed to have a high connectivity degree by PPI analysis. The results of the present study indicated that the MAPK-regulated AP1 pathway may contribute to senescence-associated disc degeneration. The DEGs, including HSP90 and CXCL5, with a high degree of connectivity may be potential targets for future investigations into molecular biomarkers.

\section{Introduction}

Back pain is a common and costly neurological ailment (1). Approximately $85 \%$ of people worldwide will experience back pain at some point in their lives, and lifetime, annual and point prevalence rates are $38.9,38.0$ and $18.3 \%$, respectively. In the United States of America, $\$ 100$ billion is spent annually on treatment for individuals that suffer with back pain (2). Chronic back pain is closely associated with degenerative alterations in intervertebral discs. Degenerative intervertebral disc changes are associated with reduced proteoglycan content, loss of bound water molecules, decreased tissue osmotic pressure and a resulting decline in the ability of the tissues to absorb biomechanical forces (3). Disc degeneration is the result of various factors, including age, mechanical stress, genetic determinants and lifestyle, which are generally considered to be its major causes. Current treatments for intervertebral disc degeneration and back pain range from conservative methods that are based on alleviating the symptoms, including back exercises to strengthen back muscles, painkillers and physiotherapy, to surgical procedures such as spinal fusion (4). However, none of these methods are completely successful. Although a number of studies have focused on the etiology of intervertebral disc degeneration (IVD), including genetics, cytokines and 
mechanical load, the underlying pathology remains to be fully elucidated $(5,6)$.

Previous studies have suggested a close association between cellular senescence and IVD; with increasing age and advancing disc degeneration, senescent nucleus pulposus (NP) cells accumulate in the NP $(7,8)$. Cellular senescence occurs when cells stop dividing; senescent cells remain viable but exhibit alterations in phenotype and gene expression patterns. For example, senescent cells may have altered responsiveness to external stimuli and may produce increased levels of matrix metalloproteinases (MMPs), which are associated with IVD. Although senescent cells may be observed in the aging/degenerating human disc, the mechanisms and signaling pathways involved in senescence and how these contribute to the process of IVD remain unknown.

The aim of the present study was to identify the differentially expressed genes (DEGs) associated with senescence in human annulus cells from degenerative intervertebral discs. DEGs were subsequently subjected to functional and pathway annotations, transcription factor (TF) enrichment analysis, construction of protein-protein interaction (PPI) networks and module analysis, to investigate the critical DEGs in the progression of senescence. The findings of the present study may provide the basis for further studies to identify novel potential hub genes associated with senescence in the development and progression of IVD.

\section{Materials and methods}

Microarray data extraction and DEG analysis. The gene expression profile dataset GSE17077, based on the platform of GPL1352 (Affymetrix Human X3P Array), was downloaded from the Gene Expression Omnibus (GEO; www.ncbi.nlm.nih. gov/geo/) database. The data were obtained by Gruber et al (9), who collected disc tissue samples from patients with degenerative disc disease undergoing surgical disc procedures. Standard laser capture microdissection techniques were used to collect senescent cells. In total, eight non-senescent cell samples and eleven senescent cell samples were harvested for mircoarray analysis.

Original CEL expression profiling data were processed into expression estimates following background correction, and quartile data normalization was performed using the robust multi-array average algorithm (10) with the default parameters in the $\mathrm{R}$ affy package (bioconductor.org/packages/release/bioc/html/affy.html) (11). Subsequently, t-tests were performed in the Linear Models for Microarray Data package (bioconductor.org/packages/release/bioc/html/limma. html) (12) to identify DEGs. Genes with $\mathrm{P}<0.05$ and $\log _{2}$ fold change (FC) $\mid>2$ were considered DEGs.

Hierarchical clustering analysis. To identify gene expression differences, hierarchical clustering analysis of DEGs was performed using MultiExperiment Viewer software version 4.9 (mev.tm4.org/\#/welcome) (13).

Functional and pathway annotations. To further investigate the functions and pathways of DEGs, the Database for Annotation, Visualization and Integrated Discovery (DAVID; version 6.7; david.ncifcrf.gov/) was used to obtain the enrichment in
Gene Ontology (GO) and Kyoto Encyclopedia of Genes and Genomes (KEGG) terms. To do this, DEGs were entered into the DAVID online tool to cluster the genes according to the GO (14) categories of cellular component (CC), biological process (BP) and molecular function (MF) (15) and KEGG pathway though the module of functional annotation with the count value $>2$ and $\mathrm{P}<0.05$.

TF enrichment analysis. To identify TFs that regulate the expression of senescence-associated DEGs, Web-based Gene Set Analysis Toolkit (WebGestalt; www.webgestalt.org/option. php) and Enrichr (amp.pharm.mssm.edu/lib/chea.jsp) were used. The adjusted enrichment $\mathrm{P}<0.001$ was selected as the threshold. Following analysis of DEGs associated with senescence by these two independent methods, the TFs identified by the two methods were selected as the key TFs.

Protein-protein interaction network construction. As proteins seldom function in isolation, it is important to understand the interaction of proteins by studying larger functional groups. The Search Tool for the Retrieval of Interacting Genes (STRING) database (string-db.org/) (16), which contains experimental and predicted interaction information, was used to annotate functional interactions between DEGs with the cut-off criterion of combined score $>0.7$. Subsequently, the interaction network was constructed using Cytoscape software version 3.3.0 (www.cytoscape.org/) (17). The total connectivity degree of each node in the network was calculated by connectivity degree analysis.

Screening for hub genes. Hub genes were identified as genes that: i) Were involved in one of the enriched pathways; ii) had a high connectivity degree ( $>5)$; and iii) were target genes of key TFs.

\section{Results}

Identification of DEGs. According to the cut-off criteria of $\mid \log _{2} \mathrm{FCl}>2.0$ and $\mathrm{P}<0.05,667$ DEGs, including 368 up- and 299 down-regulated genes, were obtained between senescent cells and non-senescent cells (Tables I and II). Of these, 41 and 18 were upregulated and downregulated by $\geq 5$-fold, respectively.

Hierarchical clustering analysis. To assess whether the 667 DEGs identified could separate senescent from non-senescent cells, hierarchical clustering analysis was performed. Clustering of conditions divided samples into two groups (Fig. 1). The eight gene expression profiles of non-senescent controls were clustered together, separated from the gene expression profiles of senescent cells.

GO functional and pathway enrichment analysis. The results of the GO enrichment analysis of DEGs based on $\mathrm{CC}, \mathrm{BP}$ and $\mathrm{MF}$ are presented in Fig. 2. According to the $\mathrm{BP}$ analysis, DEGs were primarily involved in phosphorylation, regulation of apoptosis, protein amino acid phosphorylation and regulation of programmed cell death. Based on the MF analysis, DEGs were associated with phospholipase $\mathrm{C}$ activity, purine ribonucleotide binding, ribonucleotide binding and 
phosphoinositide phospholipase $\mathrm{C}$ activity. In addition, DEGs were primarily located in the cytosol, nuclear lumen, organelle lumen and membrane-enclosed lumen.

Pathway enrichment analysis revealed that the DEGs were involved in axon guidance, natural killer cell mediated cytotoxicity, purine metabolism and the mitogen-activated protein kinase (MAPK) signaling pathway (Fig. 3).

TF enrichment analysis and transcriptional regulatory network construction. The DEGs were subjected to WebGestalt and ChEA to identify the TFs involved in their regulation. The results revealed that activator protein 1 (AP1), specificity protein 1 (SP1) and aryl hydrocarbon receptor (AHR) may serve important roles in regulating DEGs, as the AP1, SP1, AHR binding motifs were enriched in the DEGs, and Chip-seq experiments verified that AP1-, SP1-, AHR-targeted genes were enriched in the DEGs. Of these, AP1 and SP1 regulated 18 and 24 DEGs, respectively, in senescent cells (Fig. 4).

PPI network construction and module analysis. The STRING tool was used to obtain PPI associations of DEGs. The PPI network contained 248 nodes (proteins) and 399 lines (interactions) with the combined score $>0.7$ (Fig. 5). The connectivity degree of each node of the PPI network was calculated and the results of certain of these nodes are presented in Fig. 6.

Hub gene identification. Two hub genes with high connectivity degree (>5) were selected: HSP90AB1 (degree=57), encoding heat shock protein (HSP) 90, was involved in pathways in cancer and C-X-C motif chemokine (CXCL) 5 (degree=8) was involved in chemokine signaling pathway. HSP90AB1 and CXCL5 were target genes of the TFs SP1 and AP1, respectively.

\section{Discussion}

Aging is the most important single risk factor for IVD (18). Intervertebral discs undergo age-associated degenerative alterations that contribute to lower back pain; an important socio-economic problem. The link between aged degenerative discs and cellular senescence has been previously observed in human discs (19); however, the senescence-associated gene expression alterations and underlying molecular mechanisms remain unknown. The present study performed an in-depth and extensive analysis based on gene expression profiling combined with bioinformatics. Gene expression profile data from two groups (senescent and non-senescent cells) were downloaded from the GEO database and subsequently analyzed by bioinformatics methods. In total, 667 DEGs, including 368 up- and 299 down-regulated genes were screened. Hierarchical clustering analysis revealed that these 667 DEGs could separate senescent cells from non-senescent controls. The DEGs were primarily involved in axon guidance, natural killer cell mediated cytotoxicity, purine metabolism and the MAPK signaling pathway.

Transcription factors are central regulators of gene expression. Following analysis of the DEGs with ChEA and WebGestalt, the TFs AP1, SP1 and AHR were identified as potential regulators of senescence in disc degeneration. Of these, AP1, which is involved in numerous cellular processes, including proliferation, transformation and death, regulated 18
Table I. Top 15 upregulated differentially expressed genes with $\left|\log _{2}(\mathrm{FC})\right|>2$ and $\mathrm{P}<0.05$.

\begin{tabular}{lrrr}
\hline Gene & Gene ID & FC & P-value \\
\hline NCK1 & 4690 & 9.56 & 0.0001 \\
HOXB7 & 3217 & 9.28 & 0.0035 \\
HNRPDL & 9987 & 9.15 & 0.0214 \\
CNTROB & 116840 & 8.03 & 0.0001 \\
SPATA6L & 55064 & 7.98 & 0.0078 \\
EFCAB7 & 84455 & 7.94 & 0.0081 \\
HOXB9 & 3219 & 7.80 & 0.0011 \\
CYP2B6 & 1555 & 7.70 & 0.0460 \\
AHSA2 & 130872 & 7.26 & 0.0002 \\
ST3GAL5 & 8869 & 7.03 & 0.0152 \\
PPME1 & 51400 & 6.95 & 0.0019 \\
NME6 & 10201 & 6.76 & 0.0006 \\
IDS & 3423 & 6.60 & 0.0001 \\
MAN2A2 & 4122 & 6.58 & 0.0005 \\
FILIP1L & 11259 & 6.57 & 0.0033 \\
\hline
\end{tabular}

FC, fold change.

Table II. Top 15 downregulated differentially expressed genes with $\left|\log _{2}(\mathrm{FC})\right|>2$ and $\mathrm{P}<0.05$.

\begin{tabular}{lrll}
\hline Gene & Gene ID & FC & P-value \\
\hline GTPBP8 & 29083 & 0.11 & 0.0083 \\
GPATCH2 & 55105 & 0.11 & 0.0145 \\
LOC441461 & 441461 & 0.11 & 0.0126 \\
IQCC & 55721 & 0.15 & 0.0015 \\
ZNF347 & 84671 & 0.15 & 0.0017 \\
TMEM74B & 55321 & 0.15 & 0.0062 \\
F2RL2 & 2151 & 0.17 & 0.0108 \\
ATG4B & 23192 & 0.17 & 0.0045 \\
RBM26-AS1 & 100505538 & 0.17 & 0.0152 \\
UBALD1 & 124402 & 0.17 & 0.0002 \\
CSTF3-AS1 & 338739 & 0.18 & 0.0071 \\
OBSL1 & 23363 & 0.18 & 0.0224 \\
ALPK1 & 80216 & 0.18 & 0.0147 \\
LOC155060 & 155060 & 0.18 & 0.0300 \\
C1orf116 & 79098 & 0.19 & 0.0005
\end{tabular}

FC, fold change.

DEGs and SP1, which is involved in cell differentiation, growth and apoptosis, regulated 24 DEGs in the senescent cells. AP1 and SP1 have been demonstrated to promote the activation of beta1,3-glucuronosyl transferase 1, a key enzyme required for chondroitin sulfate biosynthesis, in NP cells treated with growth factors, and this process was mediated through the MAPK signaling pathway (20). Importantly, in various cell types, AP1 was highly activated in senescence $(21,22)$. Notably, 


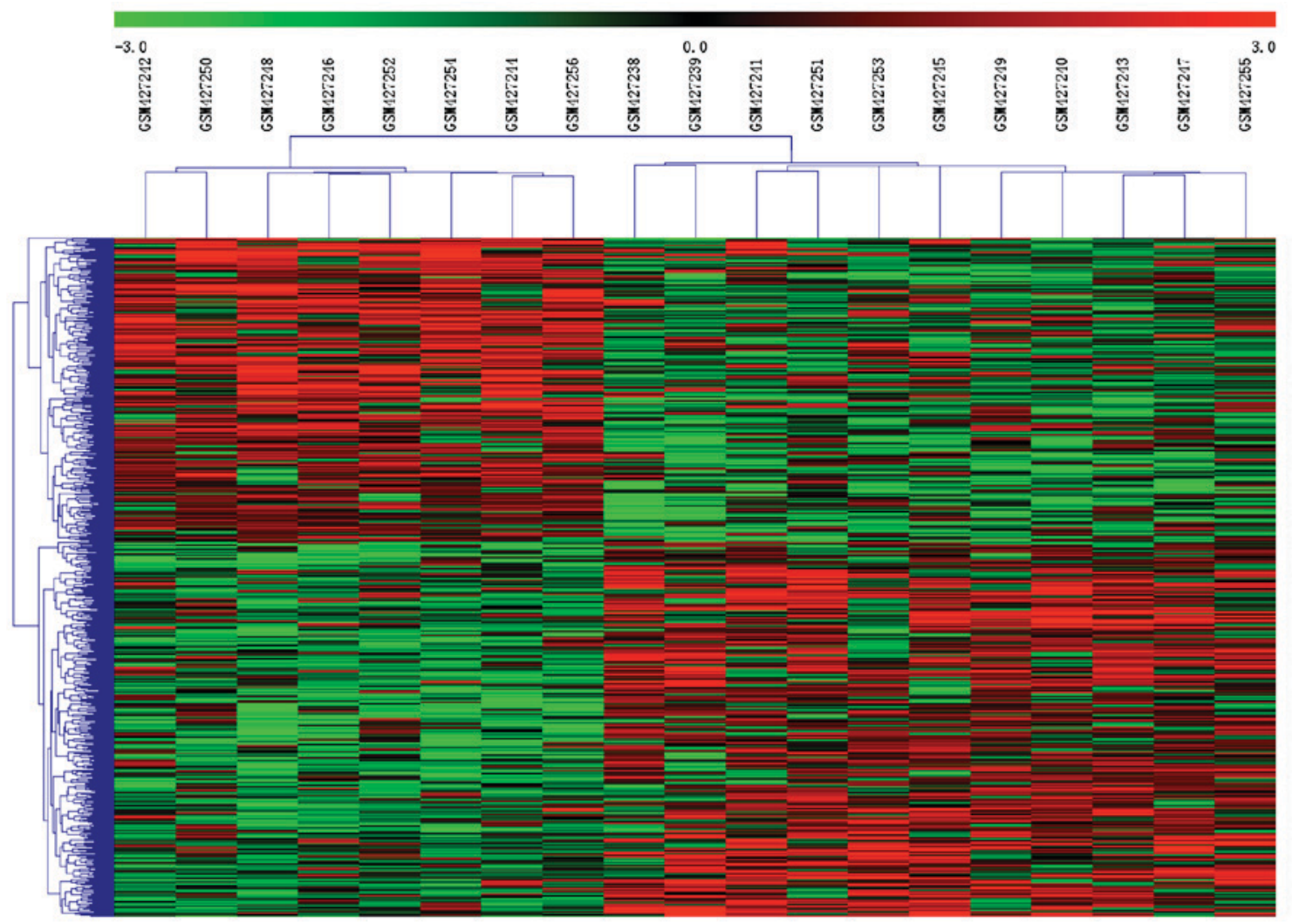

Figure 1. Hierarchical clustering of DEGs in senescent and non-senescent annulus cells. Each column represents a single sample and each row a DEG. The left 8 columns represent non-senescent cell samples and the right 11 columns represent senescent cell samples. DEG, differentially expressed gene.
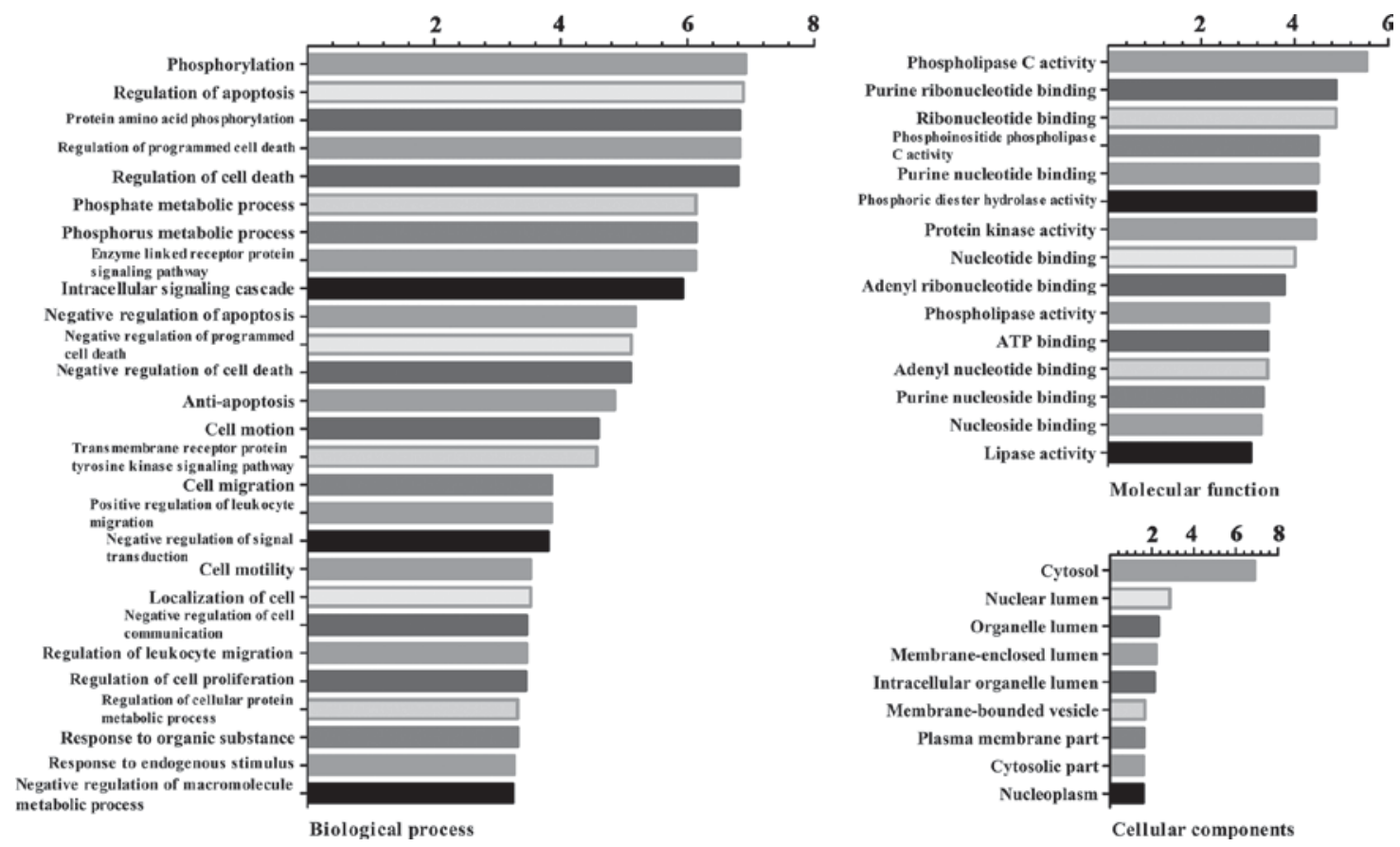

Figure 2. GO analysis of biological process, molecular function and cellular components. The vertical axis represents the GO category, and the horizontal axis represents the -lg P-value of the GO category. GO, gene ontology.

one of the enriched pathways of the DEGs identified by the present study is the MAPK signaling pathway, thus suggesting that this pathway may regulate the activation of AP1, therefore serving an important role in cell senescence and contributing to the process of disc degeneration. However, this hypothesis requires further investigation.

The present study identified two hub genes, HSP90AB1 and CXCL5. HSP90AB1 belongs to the HSP family, which has 


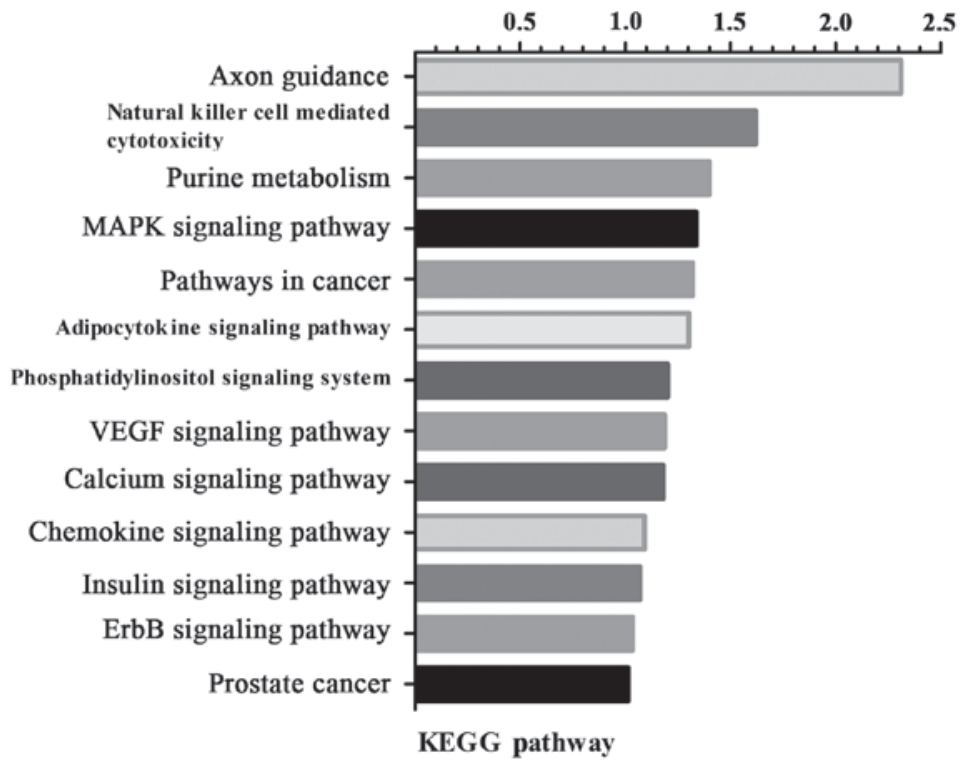

Figure 3. Enriched KEGG pathways of differentially expressed genes. The vertical axis represents the pathway category, and the horizontal axis represents the - $\lg$ P-value of the pathway category. KEGG, Kyoto Encyclopedia of Genes and Genomes.
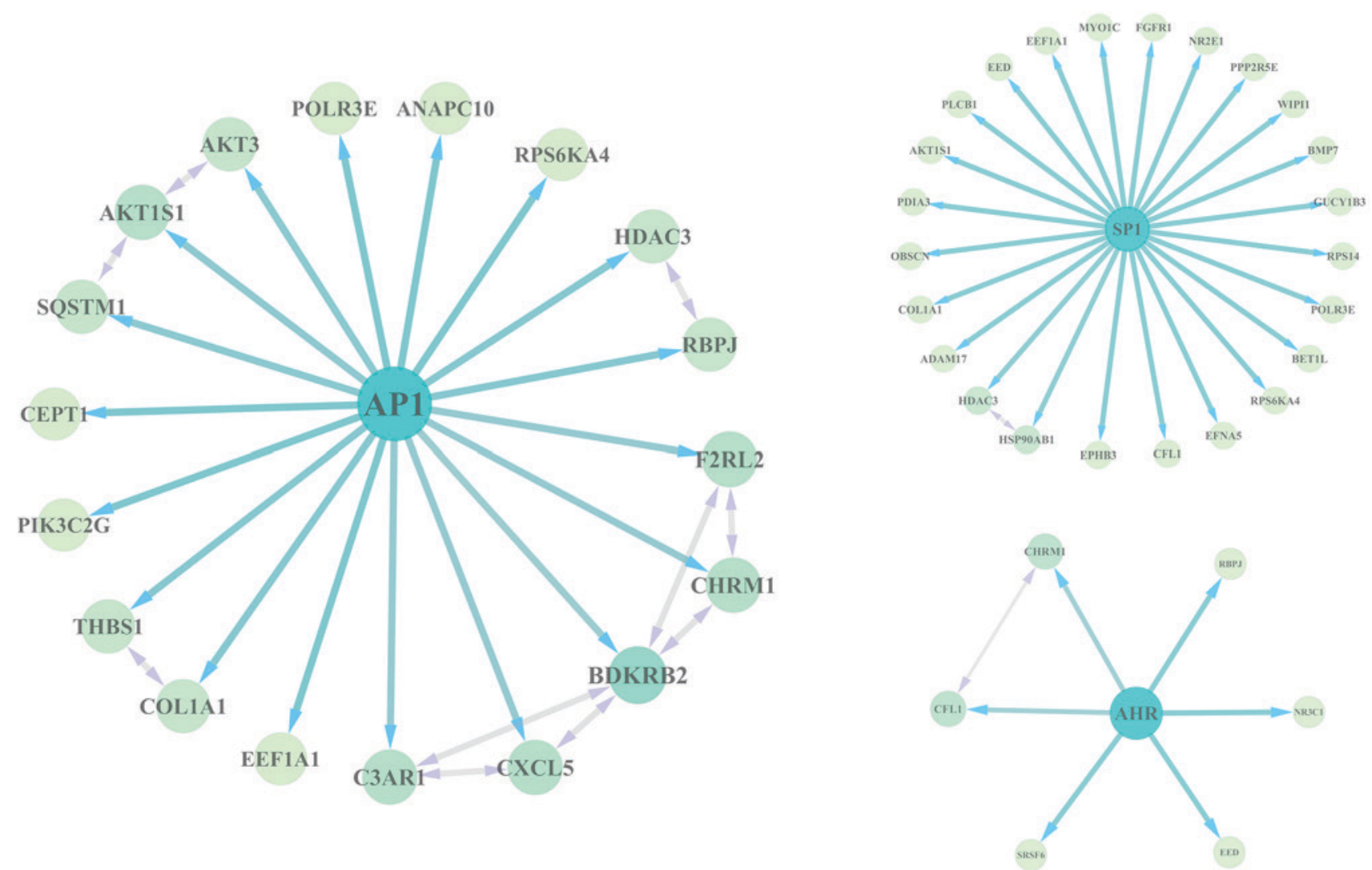

Figure 4. Regulatory network construction of TFs and their target genes within DEGs. Central blue nodes represent TFs and green nodes represent target DEGs. TF, transcription factor; DEG, differentially expressed gene; AP1, activator protein 1; SP1, specificity protein 1; AHR, aryl hydrocarbon receptor.

a number of members now recognized to be associated with cell senescence, including HSP70, which has been revealed to be downregulated in senescent annulus cells (9). Additionally, HSP90 inhibition has been demonstrated to induce cellular senescence in certain cancer cells (23). However, in contrast to previous studies, the results of the present study demonstrated that HSP90AB1, with the highest connectivity degree of 57 , was $>2$-fold greater expressed in senescent compared with non-senescent annulus cells, indicating that the function of HSP90 is cell-specific.

Chemokine expression within the IVD has been demonstrated by a number of previous reports $(24,25)$. In the present 


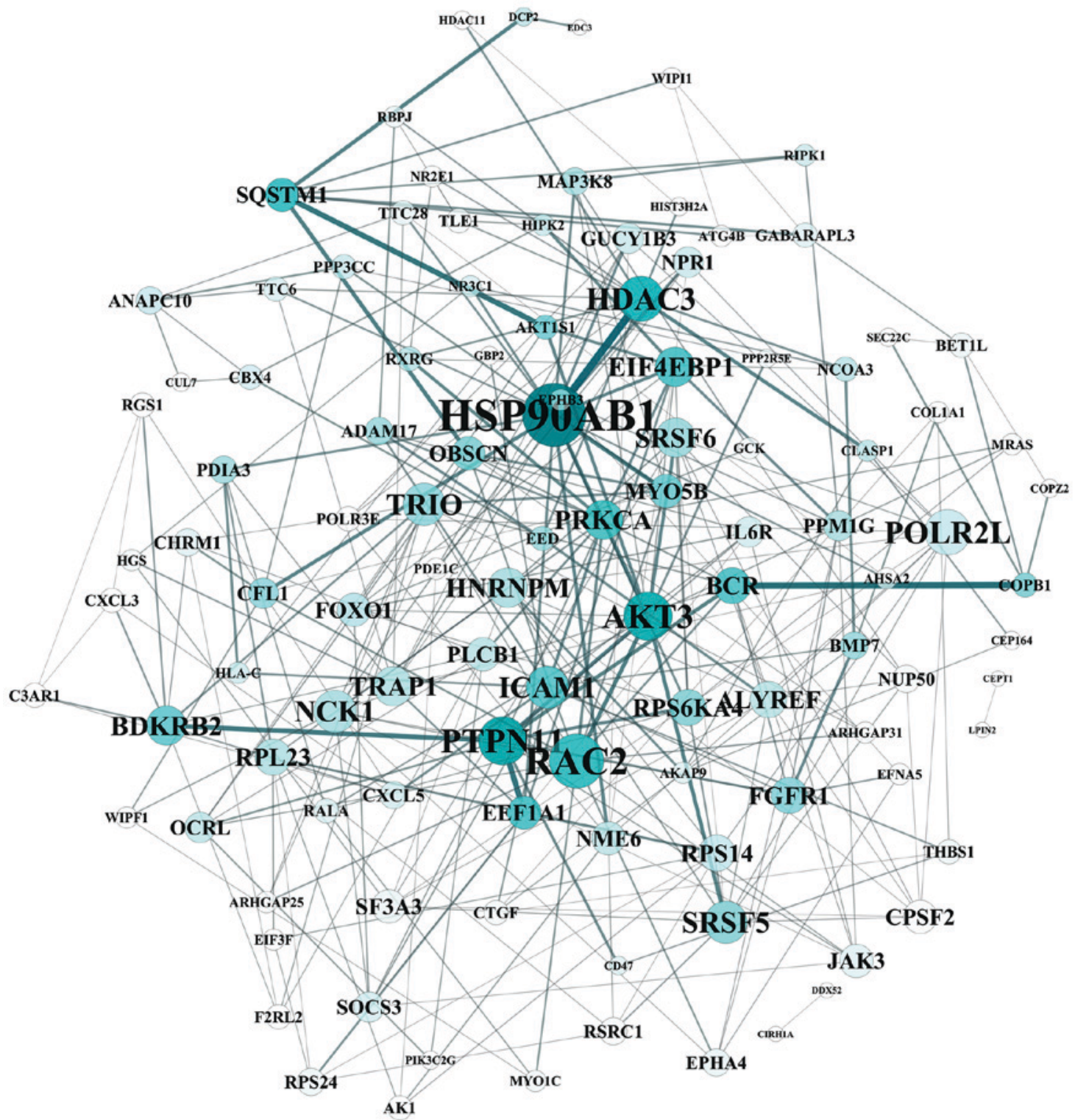

Figure 5. Protein-protein interaction network of DEGs. Light blue circular nodes represent DEGs. Increasing degree is indicated by larger nodes, labels and darker color. DEG, differentially expressed gene.

study, chemokine signal pathway was enriched among the DEGs, and chemokines including CXCL5 and CXCL3 were increased $>3$-fold in senescent compared with non-senescent cells with a high degree. Notably, CXCL5 was additionally predicted as a target gene of the TF AP1. Acosta et al (26) demonstrated that knockdown of the chemokine receptor CXCR2 inhibits senescence and that ectopic expression of CXCR2 results in premature senescence in human fibroblasts, which suggests that senescent cells activate a self-amplifying secretory network. Senescent cells may express elevated levels of extracellular matrix-degrading proteases, collagenases and MMPs, and decreased levels of the MMP inhibitor tissue inhibitor of metalloproteinases 1. A similar shift has been identified in aging and degenerated IVDs (27). Therefore, senescent disc cells may disturb extracellular matrix homeostasis via increased production of chemokines, resulting in reduced matrix synthesis and increased matrix degradation compared with non-senescent cells, eventually leading to degeneration. Additionally, the MAPK signaling pathway and the TF AP1 may be involved in this regulation.

One of the limitations of the present study is that the screened genes identified by statistically significant differences were not confirmed experimentally. That these genes are increased or decreased does not automatically imply causation, nor does it provide much insight regarding the mechanisms underlying these alterations. However, the results of the present study may provide the basis for further experimental studies.

In conclusion, the present study used microarray analysis combined with bioinformatics techniques to reveal the involvement of CXCL5 and HSP90 in the progression of senescence. In addition, the MAPK-regulated AP1 pathway may contribute to senescence-associated IVD. These findings suggested potential targets for use in the diagnosis and therapy of disc degenerative diseases. However, further studies are required to confirm this data. 


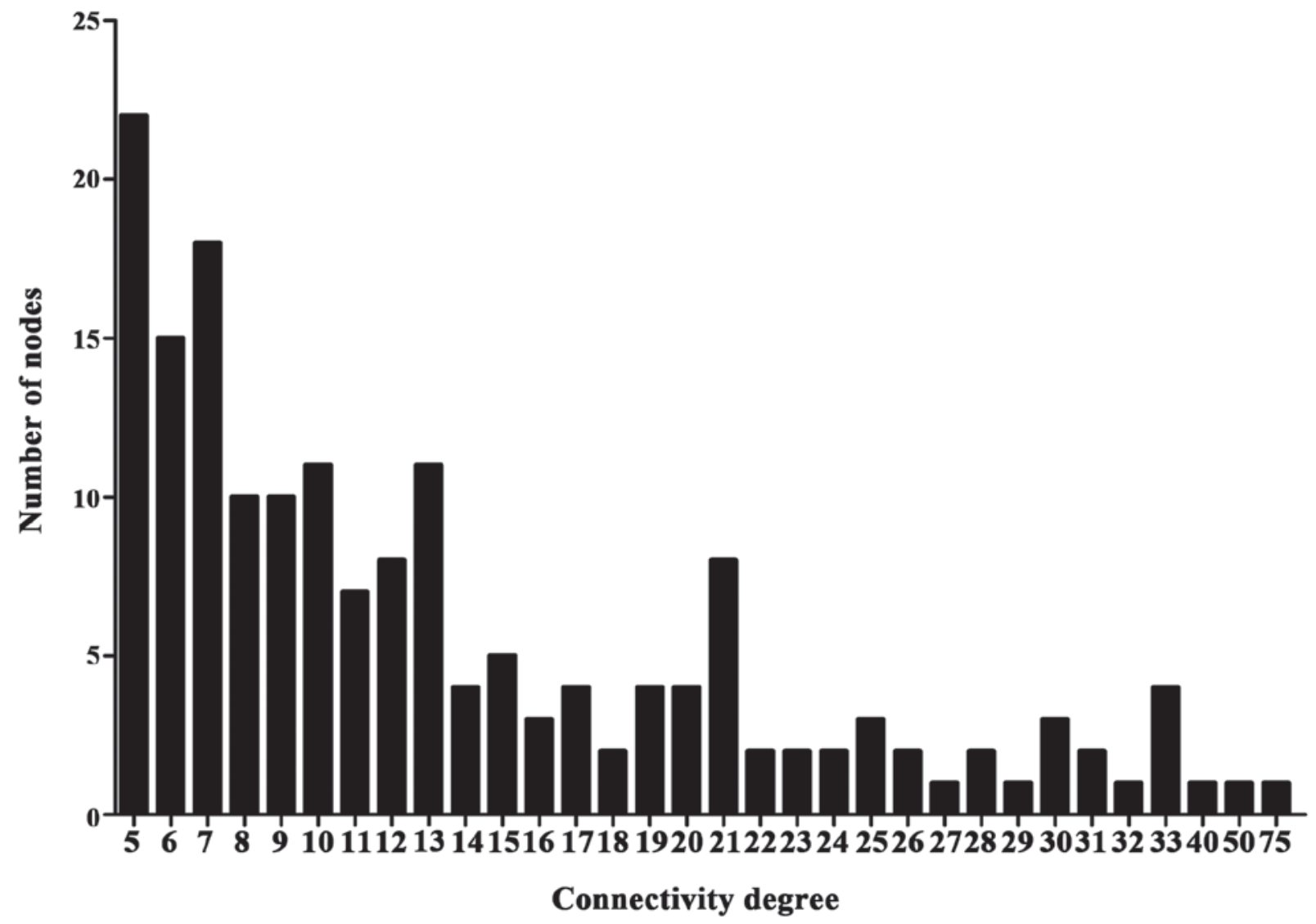

Figure 6. Connectivity degree of certain nodes in the protein-protein interaction network of differentially expressed genes.

\section{Acknowledgements}

The authors thank Dr Xinyi Liu (School of Medicine, Shanghai Jiaotong University) for technical assistance, Dr Jia Cao (Xinhua Hospital Affiliated to Shanghai Jiao Tong University School of Medicine) for data collection and Mr. Chengcai Jin (School of Medicine, Shanghai Jiaotong University) for image processing.The present study was supported by the National Natural Science Foundation of China (No. 81601920) and the Program for Tackling Key Problems in Science and Technology in Songjiang District.

\section{References}

1. Katz JN: Lumbar disc disorders and low-back pain: Socioeconomic factors and consequences. J Bone Joint Surg Am 88 (Suppl 2): S21-S24, 2006.

2. Hoy D, Bain C, Williams G, March L, Brooks P, Blyth F, Woolf A, Vos T and Buchbinder R: A systematic review of the global prevalence of low back pain. Arthritis Rheum 64: 2028-2037, 2012.

3. Luoma K, Riihimäki H, Luukkonen R, Raininko R, Viikari-Juntura $\mathrm{E}$ and Lamminen A: Low back pain in relation to lumbar disc degeneration. Spine (Phila Pa 1976) 25: 487-492, 2000.

4. Saltychev M, Eskola M and Laimi K: Lumbar fusion compared with conservative treatment in patients with chronic low back pain: A meta-analysis. Int J Rehabil Res 37: 2-8, 2014.

5. Walter BA, Korecki CL, Purmessur D, Roughley PJ, Michalek AJ and Iatridis JC: Complex loading affects intervertebral disc mechanics and biology. Osteoarthritis Cartilage 19: 1011-1018, 2011.

6. Risbud MV and Shapiro IM: Role of cytokines in intervertebral disc degeneration: Pain and disc content. Nat Rev Rheumatol 10: 44-56, 2014.
7. Gruber HE, Ingram JA, Norton HJ and Hanley EN Jr: Senescence in cells of the aging and degenerating intervertebral disc: Immunolocalization of senescence-associated beta-galactosidase in human and sand rat discs. Spine (Phila Pa 1976) 32: 321-327, 2007.

8. Kim KW, Chung HN, Ha KY, Lee JS and Kim YY: Senescence mechanisms of nucleus pulposus chondrocytes in human intervertebral discs. Spine J 9: 658-666, 2009.

9. Gruber HE, Hoelscher GL, Ingram JA, Zinchenko $\mathrm{N}$ and Hanley EN Jr: Senescent vs. non-senescent cells in the human annulus in vivo: Cell harvest with laser capture microdissection and gene expression studies with microarray analysis. BMC Biotechnol 10: 5, 2010.

10. Irizarry RA, Hobbs B, Collin F, Beazer-Barclay YD, Antonellis KJ, Scherf U and Speed TP: Exploration, normalization, and summaries of high density oligonucleotide array probe level data. Biostatistics 4: 249-264, 2003.

11. Fujita A, Sato JR, Rodrigues Lde O, Ferreira CE and Sogayar MC: Evaluating different methods of microarray data normalization. BMC Bioinformatics 7: 469, 2006

12. Ritchie ME, Silver J, Oshlack A, Holmes M, Diyagama D, Holloway A and Smyth GK: A comparison of background correction methods for two-colour microarrays. Bioinformatics 23: 2700-2707, 2007.

13. Eisen MB, Spellman PT, Brown PO and Botstein D: Cluster analysis and display of genome-wide expression patterns. Proc Natl Acad Sci USA 95: 14863-14868, 1998.

14. Ashburner M, Ball CA, Blake JA, Botstein D, Butler H, Cherry JM, Davis AP, Dolinski K, Dwight SS, Eppig JT, et al: Gene ontology: Tool for the unification of biology. The Gene Ontology Consortium. Nat Genet 25: 25-29, 2000.

15. Huang da W, Sherman BT and Lempicki RA: Bioinformatics enrichment tools: Paths toward the comprehensive functional analysis of large gene lists. Nucleic Acids Res 37: 1-13, 2009.

16. von Mering C, Huynen M, Jaeggi D, Schmidt S, Bork P and Snel B: STRING: A database of predicted functional associations between proteins. Nucleic Acids Res 31: 258-261, 2003.

17. Shannon P, Markiel A, Ozier O, Baliga NS, Wang JT, Ramage D, Amin N, Schwikowski B and Ideker T: Cytoscape: A software environment for integrated models of biomolecular interaction networks. Genome Res 13: 2498-2504, 2003. 
18. Nasto LA, Robinson AR, Ngo K, Clauson CL, Dong Q, St Croix C, Sowa G, Pola E, Robbins PD, Kang J, et al: Mitochondrial-derived reactive oxygen species (ROS) play a causal role in aging-related intervertebral disc degeneration. J Orthop Res 31: $1150-1157,2013$.

19. Le Maitre CL, Freemont AJ and Hoyland JA: Accelerated cellular senescence in degenerate intervertebral discs: A possible role in the pathogenesis of intervertebral disc degeneration. Arthritis Res Ther 9: R45, 2007.

20. Hiyama A, Gogate SS, Gajghate S, Mochida J, Shapiro IM and Risbud MV: BMP-2 and TGF-beta stimulate expression of beta1,3-glucuronosyl transferase 1 (GlcAT-1) in nucleus pulposus cells through AP1, TonEBP and Sp1: role of MAPKs. J Bone Miner Res 25: 1179-1190, 2010.

21. Sepulveda JC, Tomé M, Fernández ME, Delgado M, Campisi J, Bernad A and González MA: Cell senescence abrogates the therapeutic potential of human mesenchymal stem cells in the lethal endotoxemia model. Stem cells 32: 1865-1877, 2014.

22. Kim YJ, Hwang SH, Lee SY, Shin KK, Cho HH, Bae YC and Jung JS: miR-486-5p induces replicative senescence of human adipose tissue-derived mesenchymal stem cells and its expression is controlled by high glucose. Stem Cells Dev 21: 1749-1760, 2012.

23. Chan KC, Ting CM, Chan PS, Lo MC, Lo KW, Curry JE, Smyth T, Lee AW, Ng WT, Tsao GS, et al: A novel Hsp90 inhibitor AT13387 induces senescence in EBV-positive nasopharyngeal carcinoma cells and suppresses tumor formation. Mol Cancer 12: 128, 2013.
24. Wang J,Tian Y,Phillips KL,ChivertonN,HaddockG,BunningRA, Cross AK, Shapiro IM, Le Maitre CL and Risbud MV: Tumor necrosis factor $\alpha$ - and interleukin-1 $\beta$-dependent induction of CCL3 expression by nucleus pulposus cells promotes macrophage migration through CCR1. Arthritis Rheum 65: 832-842, 2013.

25. Kawaguchi S, Yamashita T, Katahira G, Yokozawa H, Torigoe T and Sato N: Chemokine profile of herniated intervertebral discs infiltrated with monocytes and macrophages. Spine (Phila $\mathrm{Pa}$ 1976) 27: 1511-1516, 2002.

26. Acosta JC, O'Loghlen A, Banito A, Guijarro MV, Augert A, Raguz S, Fumagalli M, Da Costa M, Brown C, Popov N, et al: Chemokine signaling via the CXCR2 receptor reinforces senescence. Cell 133: 1006-1018, 2008.

27. Roughley PJ: Biology of intervertebral disc aging and degeneration: Involvement of the extracellular matrix. Spine (Phila Pa 1976) 29: 2691-2699, 2004.

(i) (9) This work is licensed under a Creative Commons Attribution-NonCommercial-NoDerivatives 4.0 International (CC BY-NC-ND 4.0) License. 